\title{
An Experimental Study of Voting with Costly Delay*
}

\author{
Maksymilian Kwiek ${ }^{\dagger}$ \\ Department of Economics, \\ University of Southampton, \\ Southampton SO17 1BJ, \\ United Kingdom.
}

\author{
Helia Marreiros \\ University of Southampton
}

Michael Vlassopoulos

University of Southampton, IZA

November 2015

\begin{abstract}
A conclave is a voting mechanism in which a committee selects an alternative by voting until a sufficient supermajority is reached. We study experimentally welfare properties of simple three-voter conclaves with privately known preferences over two outcomes and waiting costs. The resulting game is a form of multiplayer war of attrition. Our key finding is that, consistent with theoretical predictions, when voters are ex ante heterogeneous in terms of the intensity of their preferences the conclave leads to efficiency gains relative to simple majority voting. We also compare welfare properties of a static versus a dynamic version of a conclave. When social cost of waiting is taken into account, the dynamic conclave is superior in terms of welfare than its static version.

JEL classification: C78, C92, D72, D74

Keywords: voting, supermajority, intensity of preferences, war of attrition.

${ }^{*}$ This research has received support from a University of Southampton SRDF grant and a British Academy/Leverhulme Small Research Grant. The experiment was conducted at the Social Sciences Experimental Lab of the University of Southampton and was programmed in z-tree (Fischbacher, 2007).

${ }^{\dagger}$ Corresponding author: M.Kwiek@soton.ac.uk.
\end{abstract}




\section{Introduction}

Consider a committee voting to select one of two alternatives. It is known that simple majority voting has good welfare properties in an environment with ordinal preferences (Rae, 1969). However, simple majority is not capable of expressing preference intensities. This can lead to inefficient decisions, the phenomenon that is known as "tyranny of majority". This paper focuses on a voting mechanism that we call conclave, in which the committee's decision is determined in a form of a waiting game with costly delay. Theoretical work (Kwiek, 2014a; 2014b) suggests that voting mechanisms that permit penalties, such as waiting costs, may improve welfare over simple majority. ${ }^{1}$ The aim of the current paper is to explore experimentally voting behavior and welfare performance of a simple type of such voting procedure.

Settings that share features of the voting institution we study in this paper include selecting a candidate by a hiring committee, or reaching a verdict by a trial jury. The procedure by which the Pope of the Roman Catholic Church is elected is perhaps the most fitting real-world example, and the inspiration for the name we use. Papal conclaves require a supermajority of $2 / 3$ for the decision, and many periods of voting could pass before the decision is made.

Our study relates to two strands of literature. One investigates voting mechanisms that take intensity of preferences into consideration, with an aim to improve welfare. For instance, Casella (2005) observed that linking many voting problems together may help to incentivize voters. ${ }^{2}$ Unlike these previous studies, we focus on one isolated binary decision. Secondly, the game that we study is a type of war of attrition. Hörisch and Kirchkamp (2010) and Oprea et al. (2013) experimentally investigate the benchmark case with two players and independent private information. We study a three-player committee in which two of the players have common interest in favor of one alternative. ${ }^{3}$

\section{The Environment and the Mechanism}

We consider a committee of three voters that has to choose between two alternatives. Two voters support one alternative, and the remaining one supports the another one. Committee members have private information

\footnotetext{
${ }^{1}$ Thus, we follow the classical political economy approach (for example, Rae (1969) and Azrieli and Kim (2014)), whereby monetary transfers are not allowed. We depart from this literature by introducing waiting costs.

${ }^{2}$ Other related papers are Jackson and Sonnenschein (2007), Casella et al. (2006), Engelmann and Grimm (2012), Hortala-Vallve and Llorente-Saguer (2010), Pérez-Castrillo and Veszteg (2007), Casella, Llorente-Saguer and Palfrey (2012).

${ }^{3}$ See also theoretical studies of Ponsati and Sakovics (1996) and Bulow and Klemperer (1999).
} 
about how much they value their preferred alternative.

A voting conclave is a mechanism in which an alternative is selected by voting repeatedly until a sufficient supermajority in its favor is reached. To study a tractable version of a conclave in the lab, we use a form of a waiting game, whereby voters initially support their preferred alternative, but may irreversibly cease supporting it at any time. The alternative that loses support of its last supporter is rejected by the committee, and the other alternative - the one that still has positive support - is selected. Staying in the game is costly for voters. The waiting cost is determined by a voter's exit time or the time when the waiting game stops. That is, if the exiting voter is not the last one supporting an alternative, then the waiting game continues even after her exit. However, her waiting cost is the monetary value of her actual waiting time, rather than the time when the committee reaches the decision.

The final payoff of a voter whose alternative is selected is her value of the alternative minus her waiting cost; the voter whose alternative is not selected pays her waiting cost. ${ }^{4}$

In terms of efficiency, we distinguish between two notions of welfare. Allocative (gross) utilitarian welfare is only concerned with utilities generated by the selected alternative, disregarding the waiting cost. Net welfare subtracts waiting cost from allocative welfare, treating it as social waste.

\section{$3 \quad$ Research questions}

Our first research question asks whether heterogeneity of voters' values affects the efficiency performance of the conclave. Ex ante heterogeneity measures how far the individuals' private values are from their expected value, conditional on supporting a given alternative. Low heterogeneity means that individuals' values are likely to be similar. High heterogeneity means that a randomly selected supporter is close to being indifferent, but once in a while a very high valuation may be realized. ${ }^{5}$

Our second objective is to investigate differences between the dynamic waiting game described above and its static version. In the static version, agents simply report their intended exit times and then the computer calculates the outcome. The static and the dynamic version of the game are equivalent from the strategic point of view, because voters do not get feed-

\footnotetext{
${ }^{4}$ In the experiment we assume a two-point value distribution. A symmetric equilibrium in this case involves mixed strategies. It is beyond the scope of this paper to directly assess whether voting behavior in the experiment is close to equilibrium.

${ }^{5}$ Previous theoretical work (Yoon, 2011; Kwiek, 2014a, 2014b) suggests that, as the distribution of values becomes more ex ante heterogeneous, conclave should perform better in relation to simple majority voting. Intuitively, efficiency is obtained if voters with very extreme values have a significant influence on which alternative is selected, not the ones that are almost indifferent. More stringent supermajority rule gives them that power.
} 
back about the behavior of other voters in either version. However, there is experimental evidence in the context of a classic two-player war of attrition (Hörisch and Kirchkamp, 2010), that players are less aggressive in the dynamic version. We, therefore, expect that voters will be more conservative and consequently waiting costs will be smaller in the dynamic version of the conclave.

\section{Experimental Design}

In all our treatments, we allow voters to have a two-point value distribution, low or high. We run two treatments of the static version of the game with different distributions of private values.

Treatment Static-Low - this is a static treatment with low ex ante heterogeneity of private values. Namely, a voter can be of low value $L=70$ with probability $2 / 3$, and of high value $H=160$ with probability $1 / 3$. The mean value is 100 .

Treatment Static-High - a static treatment with high heterogeneity. A voter can be of low value $L=20$ with probability $2 / 3$, and of high value $H=260$ with probability $1 / 3$. The mean value is also 100 .

In the dynamic version of the game, we only implement the high heterogeneity case. That is,

Treatment Dynamic-High - this is dynamic treatment with high heterogeneity of private values: $L=20$ with probability $2 / 3$, and of high value $H=260$ with probability $1 / 3$.

The remaining elements of the experimental design are common across treatments. Each session consists of 30 or 40 voting rounds (75-min), with random assignment of subjects into three-voter committees in each round. Each session has 15 (sometimes 12) subjects, for a total of 117 subjects. ${ }^{6}$

All values and costs are measured in Experimental Monetary Units (1 $\mathrm{EMU}=1$ penny). The sequence of events that subjects experience within each round is as follows:

- Participants are randomly assigned to a three-member committee (and are randomly rematched in every round).

- Each committee member receives a valuation, high or low.

- In a static treatment, the subjects report their intended exit costs (between 0 and $300 \mathrm{EMU}$ ). In the dynamic treatment, the subjects face a clock, which measures time from 0 to 60 seconds, and they

\footnotetext{
${ }^{6} \mathrm{~A}$ unit of observation is a committee decision.
} 
choose when to exit. Staying in the game costs 5 EMU per second, and hence the costs also varies between 0 and 300 EMU.

- The computer solves for the selected alternative and the individual waiting costs. ${ }^{7}$

In order to avoid negative payoffs, subjects receive an endowment of 900 EMU at the beginning of a session, and only three periods are randomly selected for calculating the subject's payment. The average payment was $£ 16$ including a show-up fee of $£ 4$.

\section{Results}

In both static versions of the conclave that we investigate, high valuation voters choose higher exit costs than low valuation voters. In fact, the c.d.f. of exit costs of high valuation voters first order stochastically dominates that of low valuation voters (see Figure 1). Note also that in treatment StaticHigh, high valuation voters choose higher exit costs than their counterparts in treatment Static-Low, while low types seem to select similarly in the two treatments. $^{8}$

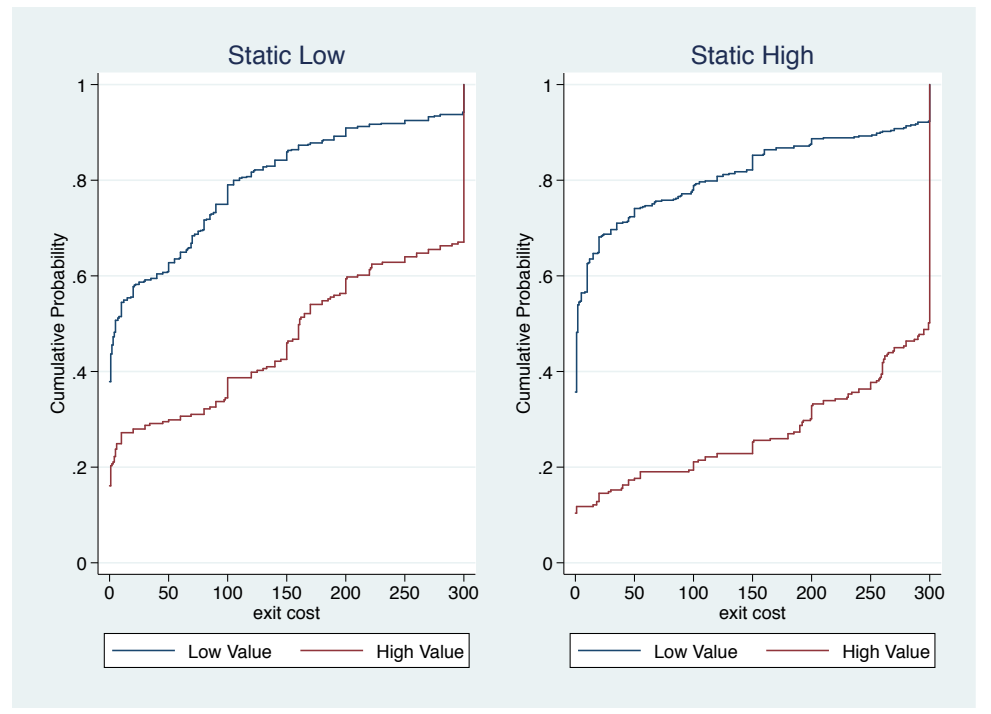

Figure 1: Cumulative probability of intended exit costs in Static versions of the conclave.

\footnotetext{
${ }^{7}$ In case of a tie, the computer randomly selected one of the alternatives and computed payments accordingly.

${ }^{8}$ Pairwise tests of equality of the distributions in Figure 1 reject equality (KolmogorovSmirnov test, $\mathrm{p}$-value $=0$ ). We can also confirm in unreported regression analysis that exit times are higher for high valuation voters controlling for periods, session and even individual fixed effects.
} 
In the Dynamic treatment, we do not always observe the exit times of all voters, as the voting game may end before all voters declared an exit time. Therefore, instead of individual exit times we present the c.d.f. of the actual ending time of the voting round in Figure 2. We can see that exit happens quite early and sooner than the static case: half of the games end at time zero, while $80 \%$ of the games end within 10 seconds. A Kolmogorov-Smirnov test of equality of distribution yields a $\mathrm{p}$-value $=0$.

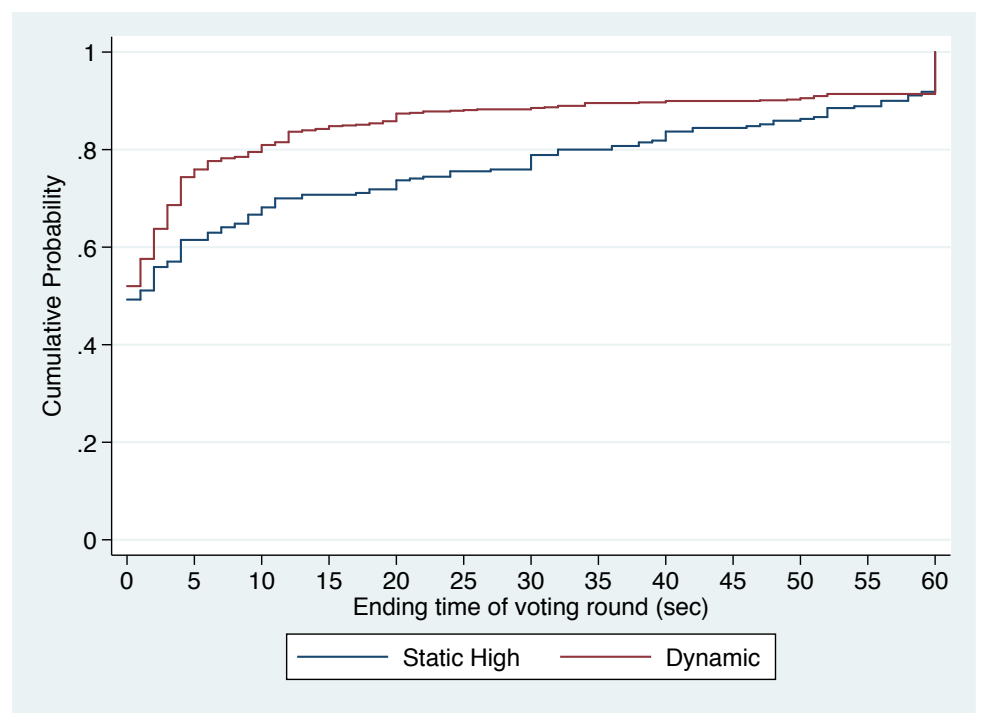

Figure 2: Cumulative probability of ending time of voting round.

We next turn attention to the efficiency of the decisions made by committees in the experiment. Table 1 summarizes the efficiency performance of conclave in each of the three treatments we consider. For reference, the table also reports the efficiency that would be achieved under simple majority voting, which we compute assuming that the alternative supported by two voters is selected. 


\begin{tabular}{lccc}
\hline \hline & Static-Low & Static-High & Dynamic-High \\
\hline Number of Subjects & 30 & 27 & 60 \\
Number of Committee decisions & 300 & 270 & 699 \\
\hline Simple Majority Voting & 198.2 & 214.2 & 205.8 \\
Conclave Allocative Efficiency & 173.5 & 221.3 & 219.6 \\
& $(88 \%)$ & $(103 \%)$ & $(107 \%)$ \\
Conclave Net Efficiency & 48.4 & 59.4 & 166.1 \\
& $(24 \%)$ & $(28 \%)$ & $(81 \%)$ \\
\hline
\end{tabular}

Table 1: Mean efficiency, in EMU. Brackets show conclave efficiency in comparison to simple majority. Note that the theoretical simple majority efficiency is 200 EMU.

We obtain the following 3 main results.

Result 1: Allocative efficiency is higher in the Static-High than the StaticLow treatment (Mann-Whitney test, $\mathrm{p}$-value=0). Moreover, simple majority is allocatively more efficient than the conclave in the StaticLow treatment (Wilcoxon signed-rank test, $\mathrm{p}$-value $=0$ ), but it is less efficient in the Static-High treatment (Wilcoxon signed-rank test, $\mathrm{p}$ value $=0.007$ )

Result 2: Static-High is similar to Dynamic-High in terms of allocative efficiency, (Mann-Whitney test, $\mathrm{p}$-value=0.6), but Dynamic-High fares better in terms of net welfare (Mann-Whitney test, $\mathrm{p}$-value $=0$ ).

Result 3: The Dynamic-High performs better than simple majority in terms of allocative efficiency (Wilcoxon signed-rank test, p-value=0).

Figure 3 shows efficiency of the dynamic conclave and of simple majority for comparison, for each type of committee configuration separately. ${ }^{9}$ We can see that the efficiency gains of the conclave arise from the configuration of values where simple majority fails to select the efficient outcome (LL-H). In this case, the dynamic conclave does substantially better than simple majority voting, while in all other cases the two mechanisms are indistinguishable.

\footnotetext{
${ }^{9}$ For example, HL-L is a committee in which the majority group has one high value and one low value voter, and the minority has a low value voter.
} 


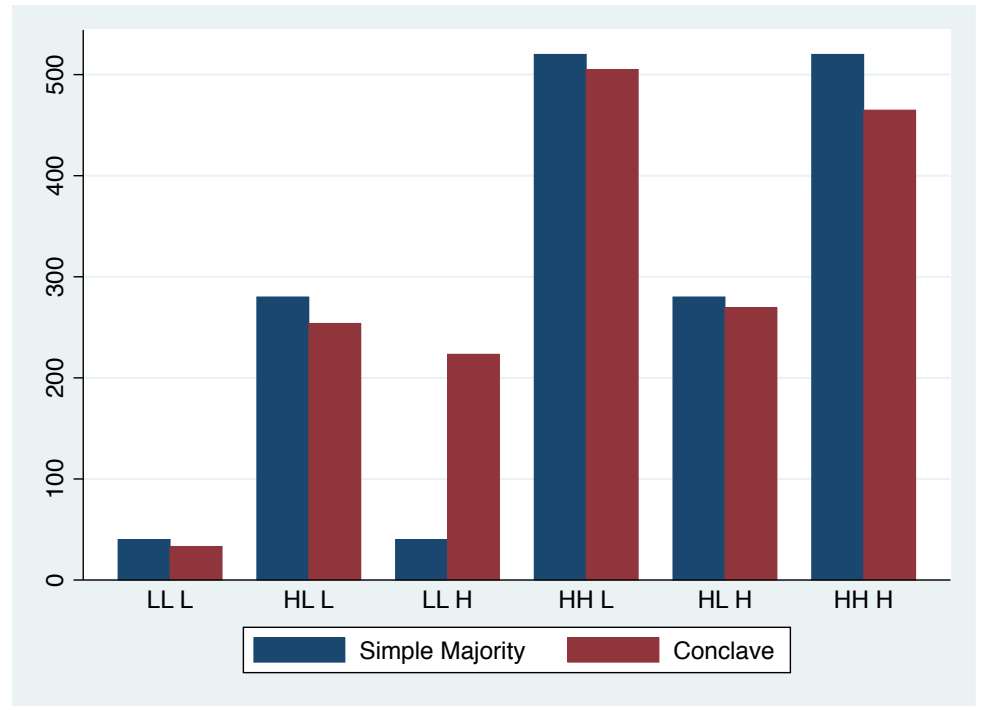

Figure 3: Allocative efficiency of Dynamic-High by committee configuration.

\section{Conclusions}

This paper reports results of experiments designed to assess the welfare performance of conclaves in comparison to simple majority voting. As expected, a more stringent supermajority is more efficient than simple majority, if agents are ex ante heterogeneous (Result 1). Our results are also consistent with an experimentally observed anomaly, whereby the dynamic war of attrition induces less aggressive behavior than its static version - despite the fact that the two are strategically equivalent (Result 2). In terms of allocative efficiency, the dynamic conclave outperforms simple majority (Result 3).

\section{References}

[1] Azrieli Y. and S. Kim, (2014) Pareto Efficiency And Weighted Majority Rules, International Economic Review, 55(4).

[2] Bulow, Jeremy, and Paul Klemperer. (1999), The Generalized War of Attrition. American Economic Review, 89(1): 175-189.

[3] Casella, Alessandra, (2005), Storable votes, Games and Economic Behavior, 51, 391-419.

[4] Casella Alessandra, Andrew Gelman, Thomas R. Palfrey, (2006), An experimental study of storable votes, Games and Economic Behavior $57,123-154$. 
[5] Casella, Alessandra, Aniol Llorente-Saguer, and Thomas R. Palfrey. (2012), Competitive Equilibrium in a Market for Votes, Journal of Political Economy, 120(4), 593-658.

[6] Engelmann, Dirk, and Veronika Grimm, (2012), Mechanisms for Efficient Voting with Private Information about Preferences, The Economic Journal, 122, 1010-1041.

[7] Fischbacher, Urs. (2007) 'z-Tree: Zurich Toolbox for Ready-Made Economic Experiments. Experimental Economics, 10, 171-8.

[8] Hörisch H., and O. Kirchkamp, (2010), Less fighting than expected experiments with war of attrition and all-pay auctions, Public Choice, 144(1), 347-367.

[9] Hortala-Vallve, Rafael, and Aniol Llorente-Saguer, (2010), A simple mechanism for resolving conflict, Games and Economic Behavior, 70, $375-391$.

[10] Jackson, Matthew O. and Sonnenschein, Hugo F, (2007), Overcoming Incentive Constraints by Linking Decisions, Econometrica, 75(1), 241-257.

[11] Kwiek, Maksymilian, (2014a), Conclave, European Economic Review, $70(\mathrm{C}), 258-275$.

[12] Kwiek, Maksymilian, (2014b), Efficient voting with penalties, Discussion Paper, 1419, Economics, University of Southampton.

[13] Oprea Ryan, Bart Wilson and Arthur Zillante, (2013), War Of Attrition: Evidence From A Laboratory Experiment On Market Exit, Economic Inquiry, 51(4), 2018-2027

[14] Pérez-Castrillo, David, and Róbert F. Veszteg, Choosing a common project: Experimental evidence on the multibidding mechanism, Journal of Economic Behavior and Organization, 63(3), 394-411.

[15] Ponsati Clara, Jozsef Sakovics, (1996), Multiperson Bargaining over Two Alternatives, Games and Economic Behavior, 12(2), 226-244.

[16] Rae, Douglas W. (1969), Decision-Rules and Individual Values in Constitutional Choice, The American Political Science Review, 63(1), 40-56.

[17] Yoon, K. (2011), Optimal mechanism design when both allocative inefficiency and expenditure inefficiency matter, Journal of Mathematical Economics, 670-676. 